\title{
Direct Gene Transfer for the Understanding and Treatment of Human Disease
}

\author{
GREGORY E. PLAUTZ, ${ }^{a, b}$ ELIZABETH G. NABEL, ${ }^{c}$ \\ BERNARD FOX, ZHI-YONG YANG, ${ }^{c, d, g}$ \\ MICHELE JAFFE, $, d, g$ DAVID GORDON, ${ }^{f}$ \\ ALFRED CHANG,' AND GARY J. NABEL ${ }^{c, d, \theta}$ \\ Departments of ${ }^{b}$ Pediatrics and Communicable Diseases, 'Internal \\ Medicine, "Biological Chemistry, 'Surgery, and fPathology, and \\ sHoward Hughes Medical Institute \\ University of Michigan Medical Center \\ Ann Arbor, Michigan 48109-0684
}

\section{INTRODUCTION}

Gene transfer offers the potential to analyze the effects of recombinant genes in cells and organisms. Most applications of gene transfer are performed on cells in vitro. Recently, however, recombinant genes have been introduced directly into tissues in vivo. In this laboratory, viral and nonviral vectors have been introduced through intraarterial catheters to achieve recombinant gene expression in localized segments of blood vessels. The effects on arteries induced by localized expression of several genes with mitogenic and angiogenic effects have been analyzed in vivo. Through intravascular gene transfer, genes involved in the pathogenesis of atherosclerosis are being defined in clinically relevant animal models of disease. Identification of genes that can regulate proliferation of vascular cells and alter their thrombogenicity in vipo may provide gene-based strategies to ameliorate this disease.

Cancer cells are derived from normal precursors, which undergo a series of genetic alterations in successive clones of cells, resulting in uncontrolled proliferation and metastasis. Abnormal gene products synthesized by neoplastic cells can potentially generate novel tumor antigens. Appropriate stimulation of the immune system can induce a cell-mediated immune response directed against tumor antigens, and the transfer of immunostimulatory genes into nonimmunogenic tumor cells can stimulate antitumor responses in animals that are protective against the nontransduced tumor cells upon rechallenge. We utilized direct gene transfer to introduce an allogeneic class I major histocompatibility complex (MHC) gene into tumors in vivo. Expression of this recombinant gene within tumors stimulated a cell-mediated antitumor immune response. Unlike

${ }^{a}$ Address for correspondence: Dr. Gregory E. Plautz, Department of Pediatrics and Communicable Diseases, University of Michigan Medical Center, MSRB I Room A510, 1150 West Medical Center Drive, Ann Arbor, Michigan 48109-0684. 
transduction of tumor cells in vitro, and subsequent inoculation into a nontumor-bearing host, direct gene transfer allows modifications of established tumors. Thus, strategies to augment the immune response to preexisting tumors can be evaluated, providing a more realistic model for clinical applications in human malignancies.

In this paper, we review the application of direct gene transfer to the study of vascular biology and its use for provision of immunotherapy in malignancy. Direct gene transfer with DNA liposome complex is less costly and time consuming than cell-mediated gene transfer. Moreover, the stability of liposome and DNA preparations and the ease with which they can be prepared and administered to recipients could facilitate their use in clinical therapy.

\section{METHODS OF DIRECT GENE DELIVERY}

Direct gene transfer is a useful method to modify endogenous cells in vipo to study the effects of recombinant gene expression. Several methods of gene transfer in vivo have been demonstrated. Viral vectors, including retroviruses, ${ }^{1-3}$ adenoviruses, ${ }^{4-6}$ adeno-associated viruses, ${ }^{7}$ and herpes viruses ${ }^{8-10}$ have been modified to express recombinant genes and eliminate their ability to replicate. Direct injection of DNA into certain cell types such as skeletal or cardiac myocytes and melanoma has allowed expression of recombinant genes. ${ }^{11-14}$ DNA forms a complex with cationic liposomes that improves the efficiency of recombinant gene transfer into cells in vivo. $2,15-19$

Cationic liposomes exhibit features that significantly improve their efficacy for in vivo gene transfer. They can be prepared in large batches by sonication to form particles of approximately $250-\mathrm{nm}$ diameter that are stable in aqueous solution for months. Through an electrostatic interaction, the lipids condense with the DNA to efficiently encase it. Liposomes interact with the cell surface, allowing the DNA to traverse the plasma membrane of target cells. The DNA is subsequently transferred to the nucleus to direct transcription through incompletely defined mechanisms. ${ }^{20}$ Cationic liposomes can be significantly inactivated by serum. ${ }^{21}$ Therefore, transduction of cells is most effective at the site of delivery. Two cationic liposome preparations have been used in this laboratory for site-specific gene transfer. Lipofectin (Bethesda Research Laboratories, Gaithersburg, MD), a mixture of DOTMA (N-[2,3-dioleyloxy)propyl]-N,N,Ntrimethylammonium chloride) and DOPE (dioleoyl phosphatidylethanolamine), ${ }^{22}$ has been used to deliver recombinant genes into vascular cells in peripheral ${ }^{2,23}$ and coronary ${ }^{24}$ arteries in vivo. Another cationic liposome preparation, containing DC-cholesterol ( $3 \beta\left[N-\left(N^{\prime} N^{\prime}\right.\right.$-dimethylaminoethane)-carbamoyl] cholesterol) and DOPE, ${ }^{25}$ has also provided efficient gene transduction of vascular cells and malignant tumors ${ }^{17}$ in vipo.

Several features of liposome-mediated gene transfer facilitate its application to direct gene transfer in vipo. In contrast to retrovirus-mediated gene transfer, replication of the recipient cell is not necessary. Therefore, terminally differentiated somatic cells can serve as targets. Also, unlike viral vectors, there are few constraints on vector size or transcriptional signals, and a variety of tissue-specific 
enhancer/promoter elements or polycistronic vectors may be used. DNA liposome complexes form rapidly in aqueous solution without extensive preparation, permitting rapid analysis of a series of vectors for activity in vitro or in vipo. The DC-cholesterol preparation is nontoxic over a wide range of concentrations in vitro and is metabolized in vipo. ${ }^{25}$ Extensive toxicity testing has demonstrated the DC-cholesterol liposome preparation to be safe in vivo. $26,27,44$

\section{SITE-SPECIFIC GENE TRANSFER INTO VASCULAR CELLS IN VIVO}

To introduce recombinant genes into specific target tissues in vivo, we delivered retroviral vectors or DNA liposome complexes to anatomically localized sites. Initially, vascular cells were transduced with recombinant genes in vipo, using retroviral vectors or DNA liposome complexes instilled into localized arterial segments. In a porcine model, a segment of the iliofemoral artery was isolated from the systemic circulation using a double balloon catheter. The space between the two balloons was flushed to remove residual serum, and a concentrated suspension of amphotropic Moloney murine leukemia viral vector or DNA liposome complexes was instilled in the arterial segment for thirty minutes prior to removal of the catheter. Recombinant gene expression was present in endothelial cells, vascular smooth muscle cells, and adventitial cells in arteries transduced with the marker gene $\beta$-galactosidase. Recombinant gene expression decreased with time but was clearly present up to 21 weeks following retroviral gene transfer and was not detected in the downstream arterial segment or in other tissues by PCR analysis. ${ }^{2}$

Intraarterial direct gene transfer can elucidate the pathologic response to recombinant growth factor gene expression in normal and injured arteries. The endothelial cell growth factor fibroblast growth factor-1 (FGF-1) induces endothelial cell proliferation in vitro and is angiogenic in vipo. Because FGF-1 lacks a classical signal sequence, the study of its effects as an extracellular protein has been difficult. A chimeric gene, in which the signal sequence from the FGF-4 gene was fused to the coding sequence of FGF-1, was introduced directly into localized segments of arteries in vipo. This plasmid, complexed with Lipofectin ${ }^{\oplus}$, was transfected into porcine arteries at low instillation pressure $(150 \mathrm{mmHg})$ to minimize trauma to the vessel. Presence of the introduced DNA was confirmed 21 days after transfection by PCR, and protein expression was confirmed by immunohistochemistry. The secreted FGF-I gene induced intimal proliferation, and in several vessels capillary formation was observed in the neointima. ${ }^{18}$ Such angiogenesis has been observed in atherosclerotic plaques; however, mechanisms to explain this observation have previously been lacking. Because of its effects as an angiogenic factor, this FGF-1 vector could potentially be useful to induce neovascularization of ischemic tissue following intravascular delivery into affected tissue.

Platelet-derived growth factor (PDGF) B chain has also been implicated in the generation of atherosclerosis. This growth factor is a potent mitogen for vascular smooth muscle cells, but does not induce proliferation of endothelial 
cells. To analyze the response to this growth factor in vivo, a plasmid expression vector encoding the human PDGF B gene was introduced into porcine arteries using Lipofectin $B$ at low instillation pressures. ${ }^{19}$ The expression of introduced DNA was confirmed by PCR analysis of recombinant PDGF B mRNA. Immunohistochemistry revealed human $\mathrm{PDGF} B \mathrm{BB}$ protein expression in the intima and media but not in control arteries transduced with $\beta$-galactosidase. Quantitative morphometry of intimal and medial thickness demonstrated a sevenfold increase in the intimal-to-medical ratio compared to $\beta$-galactosidase-transduced vessels. Unlike the FGF-1-transduced arteries, the intimal thickening was not associated with neocapillary formation. These studies provide a method to examine the effects of recombinant growth factor gene expression on vascular physiology in normal and injured arteries. Such studies also provide a model to investigate inhibition of intimal proliferation using growth factor or receptor antagonists.

To analyze the response of the immune system to a foreign protein expressed on endogenous vascular cells, a human MHC gene, HLA-B7, was introduced into the iliofemoral artery of pigs using retroviral vectors or DNA liposome complexes. ${ }^{23}$ Both vectors provided expression of HLA-B7 protein within vascular cells documented by PCR analysis of DNA and mRNA and by immunohistochemistry. Expression of the foreign histocompatibility antigen by vascular cells induced an intense inflammatory response in recipient animals, consisting of a mononuclear cell infiltrate in the adventitia and media. The inflammatory response was present by 11 days after gene transfer, peaked at 17 to 21 days, and had subsided by 75 days. Some neointimal thickening, as is seen following chronic inflammation, was noted in the transduced arteries. Further studies determined that cytotoxic $T$ lymphocytes specific for the foreign antigen were generated in these animals. Control arteries transduced with the $\beta$-galactosidase gene did not develop inflammatory histologic changes or an immune response. The histologic and immunologic responses induced by HLA-B7 were similar for both retroviral and liposome-mediated gene transfer, confirming that each method can provide appropriate and biologically active gene expression. This study also demonstrated that expression of a single foreign MHC gene by normal cells could induce a significant localized immune response.

\section{DIRECT GENE TRANSFER OF ALLOGENEIC CLASS I MHC GENES INTO TUMORS}

The induction of a specific cell-mediated response generated by intravascular transfer of a foreign class I MHC gene suggested that this approach could be applied to the immunotherapy of malignancy. Previous studies using cell-mediated gene transfer demonstrated that genetic modification of tumor cells can increase their immunogenicity and stimulate a specific antitumor response. ${ }^{28-38}$ To determine whether a therapeutic immune response could be generated against preexisting macroscopic tumors, we introduced an allogeneic class I MHC gene by intratumoral injection of DNA liposome complexes or retroviral vectors. MHC alleles are highly polymorphic, and most of the variation between 
alleles is concentrated in residues that comprise the peptide binding portion of the molecule ${ }^{39}$ Consequently, transplanted tissue that expresses allogeneic MHC molecules is highly immunogenic, presumably due to the altered shape of the $\mathrm{MHC}$ in the peptide binding region. A high precursor frequency of $\mathrm{CD8}^{+}$ lymphocytes capable of reacting against allogeneic cells exists. ${ }^{40,41}$ Therefore, introduction of an allogeneic class I MHC gene into tumors should stimulate a strong immune response locally.

The murine class I MHC gene $\mathrm{H}-2 \mathrm{~K}^{\mathrm{s}}$ was introduced into tumors in vivo by direct intratumoral injection of a DNA liposome complex using DC-cholesterol liposomes. A weakly immunogenic murine tumor line CT26, a colon adenocarcinoma with the $\mathrm{H}-2^{\mathrm{d}}$ haplotype, was inoculated subcutaneously in BALB/c mice. Approximately 14 days later, when tumors were 5-8 $\mathrm{mm}$ in diameter, the tumors were transduced with the $\mathrm{H}-2 \mathrm{~K}^{\mathrm{s}}$ gene. Lymphocytes harvested from these animals 14 days after gene transfer demonstrated lytic activity against CT26 targets expressing H-2 $\mathrm{K}^{s}$. More importantly, cytotoxic lymphocytes reactive against the parental nontransduced CT26 cells were present, demonstrating the generation of an immune response to other tumor antigens. Injection of a control gene $\beta$-galactosidase complexed with the liposomes did not induce cytolytic $T$ cells. Cytolytic activity against both transduced and parental tumor cells could be blocked by antibody against $\mathrm{CD8}^{+}$but not $\mathrm{CD}^{+}$lymphocytes. This immune response was not protective in this rapidly growing murine tumor model because the tumors were relatively large at the time of gene transfer and grew progressively during the generation of the antitumor response. Experiments using intratumoral transfer of the $\beta$-galactosidase gene suggest that the efficiency of gene transfer was approximately $1 \%$.

Significant attenuation of tumor growth and, in some cases, complete regression of existing tumors were achieved following $\mathrm{H}-2 \mathrm{~K}^{\mathrm{s}}$ gene transfer when mice were presensitized to the $\mathrm{H}-2 \mathrm{~K}^{\mathrm{s}}$ gene. Mice were preimmunized against $\mathrm{H}-2 \mathrm{~K}^{\mathrm{s}}$ by intraperitoneal injection of either irradiated tumor cells expressing $\mathrm{H}-2 \mathrm{~K}^{s}$ or of splenic lymphocytes from an $\mathrm{H}-2^{\mathrm{s}}$ mouse strain. Two histologic types of tumors, the colon adenocarcinoma CT26 and the fibrosarcoma MCA 106 from two different mouse strains, $\mathrm{BALB} / \mathrm{c}$, and $\mathrm{C57BL} / 6$, respectively, responded to tumor transduction with the $\mathrm{H}-2 \mathrm{~K}^{s}$ gene. This study demonstrated that the immunologic response to $\mathrm{H}-2 \mathrm{~K}^{\mathrm{s}}$ was not strain specific or tumor specific.

The antitumor response was mediated by $\mathrm{T}$ lymphocytes. The growth of CT26 or MCA 106 tumors in nude mice treated with the $\mathrm{H}-2 \mathrm{~K}^{\mathrm{s}}$ gene was identical to that of $\beta$-galactosidase-treated mice or untreated controls. The immune response to $\mathrm{H}-2 \mathrm{~K}^{s}$ gene transfer was abrogated by depletion of $\mathrm{CD} 8^{+} \mathrm{T}$ cells, but not by depletion of $\mathrm{CD} 4^{+}$cells. Direct gene transfer of the $\mathrm{H}-2 \mathrm{~K}^{\mathrm{s}}$ gene into the poorly immunogenic murine melanoma, B 6BL6, in mice generated tumor-reactive lymphocytes in tumor-draining lymph nodes. When stimulated ex vivo with anti-CD3 mAb for two days followed by culture in low-dose IL-2 $(10 \mathrm{U} / \mathrm{ml})$ for three days, these effector cells had no direct cytolytic activity against B16BL6 targets. However, adoptive transfer of these lymphocytes to mice bearing B16BL6 lung metastases resulted in a marked decrease in lung metastases in comparison to a control gene. ${ }^{42}$ These studies demonstrate that 
actively growing macroscopic tumors can be transduced with recombinant genes in vivo by intratumoral gene transfer using DNA liposome complexes. Moreover, an appropriate immunologic response can be generated against an allogeneic MHC protein expressed within actively growing tumors, and this strong immune response can stimulate a host immune response to weak tumor antigens. Appropriate augmentation of this antitumor immune response can mediate regression of preexisting tumors.

\section{IMMUNOTHERAPY OF HUMAN MALIGNANCY BY DIRECT GENE TRANSFER: PRECLINICAL TOXICITY ANALYSIS}

To determine whether recombinant genes can be delivered safely in vivo using DNA liposome complexes, acute and long-term toxicity studies were performed in animal models. DC-cholesterol liposomes complexed with DNA were injected into mice intravenously or intratumorally, and the distribution of DNA was determined by PCR analysis of organs two to three weeks after gene transfer. The plasmid DNA was detected in heart and lung tissue in most cases and occasionally in kidney when mice received intravenous DNA liposome complexes. Intratumoral injection resulted in isolation of plasmid in tumor tissue and occasionally in heart, lung, and spleen. There were no histopathologic findings in the organs of any animals, including those containing plasmid DNA. In these instances, DNA was present at a very low level. Analysis of serum enzymes did not reveal evidence of liver, heart, or kidney dysfunction. Because of the finding of injected DNA in heart tissue, electrocardiography was performed during and after liposome injection. These electrocardiograms were all normal. ${ }^{26}$

Expression of a foreign class I MHC gene on normal tissues could potentially induce autoimmunity. Mice that received the allogeneic MHC gene all generated a specific cytolytic T lymphocyte (CTL) response to the foreign gene product; however, there was no histopathologic or serologic evidence of organ toxicity. ${ }^{27}$ It is likely that immunologic tolerance to normal tissue prevented immune mediated damage from the low levels of allogeneic gene expression in these tissues following intratumoral gene transfer. Intravenous, intraperitoneal, or subcutaneous injection of the allogeneic MHC gene complexed with liposomes did not induce a detectable pathology.

The potential for inadvertent modification of germ line tissue by recombinant gene is a concern for in vivo gene therapy. To address this question, 42 rabbits and pigs were treated with intravenous or intraarterial injection of DNA liposome complexes. Transfected DNA was never isolated from gonadal tissue by PCR, despite its presence in the transduced artery segments and occasionally in other organs. ${ }^{27}$ These studies demonstrate that direct gene transfer and expression of recombinant genes, including a foreign $\mathrm{MHC}$ gene, do not induce generalized autoimmunity to normal tissue, or detectable gene transmission to germ cells. Direct gene transfer using DNA liposome complexes therefore appeared suitable for human gene therapy. 


\section{DIRECT GENE THERAPY IN HUMAN CANCER}

A phase I/II human gene therapy trial to provide immunotherapy for malignancy by direct intratumoral injection of an allogeneic MHC gene HLA-B7 complexed with DC-Chol liposomes was approved by the Recombinant DNA Advisory Committee in January $1992 .{ }^{43}$ Patients with metastatic melanoma receive injections of the HLA-B7 gene into subcutaneous tumor nodules, with four study groups to receive escalating doses of the DNA liposome complex. The presence of transfected DNA, transcription of mRNA, and HLA-B7 protein expression are being analyzed after each injection in cells obtained from tumor biopsies. The presence of an immunologic response to HLA-B7 and autologous tumor cells are being studied using peripheral blood lymphocytes and tumorinfiltrating lymphocytes. ${ }^{44}$ The objectives of this ongoing clinical trial are: (1) to establish a safe and effective dose to introduce a recombinant gene, HLA-B7, into tumors by DNA liposome transfection; (2) to confirm expression of this class I MHC gene by tumor cells following direct gene transfer in vipo; and (3) to analyze the immune response against this antigen and other tumor antigens.

This protocol represents the first use of direct gene transfer into humans in vivo and is the first application of a nonviral vector-liposome-mediated gene transfer. ${ }^{44}$ The stability of the DNA and liposomes coupled with the ease with which they can be reconstituted and administered to patients, provide the potential for such treatment to be provided in a generalized outpatient setting. Because most of the DNA is maintained extrachromosomally following liposome-mediated gene transfer, this approach would not provide permanent correction for genetic disorders in proliferating cells. However, repeated treatments might provide sustained correction of genetic diseases at sufficient levels to ameliorate symptoms. Applications of gene therapy to many acquired disorders or to provide immunotherapy for malignancy would require only transient expression of recombinant genes. Most gene therapy models and human protocols rely on modification of target cells ex vivo. This involves considerable effort and time, and the expense of large-scale dedicated tissue culture facilities. Additionally, ex vivo gene transfer would not be applicable in situations where target cells fail to grow in culture, as commonly occurs with many somatic cells and neoplastic cells. Direct gene transfer into patients avoids many of the disadvantages of ex vivo gene therapy and minimizes delays in the initiation of treatment. Anatomically localized delivery of DNA liposome complexes directly into the parenchyma of tissues or through intravascular catheters into the vascular bed can provide site-specific expression of recombinant genes in vivo.

\section{SUMMARY}

Direct gene transfer has been used to develop molecular genetic interventions for acquired diseases in several animal models. Through the use of intravascular catheters or anatomically localized injection of DNA liposome complexes, specific tissues can be transduced with recombinant genes. Several promising applications of this method for the study of vascular biology have been demon- 
strated by direct gene transfer into arteries in vivo. Delivery, via catheter, of genes that modulate the thrombogenic or proliferative properties of vascular cells may someday provide therapy for stenotic lesions of atherosclerosis or following angioplasty. Cancer is another acquired disorder in which direct gene transfer may improve the efficacy of treatment. Introduction of class I MHC or cytokine genes with antitumor or immunostimulatory effects have demonstrated promise in animal models. Direct transfer of an allogeneic class I MHC gene into tumors in vivo induces a $\mathrm{CD8}{ }^{+} \mathrm{CTL}$ response against weak antigens on poorly immunogenic tumors. The efficacy of this antitumor response can be augmented to induce regression of actively growing established tumors. Additional strategies, such as intratumoral delivery of combinations of multiple cytokine and $\mathrm{MHC}$ genes, may serve to improve the antitumor response. A clinical gene therapy protocol is underway to analyze the safety and efficacy of DNA liposomemediated gene transfer in humans. Development of improved gene delivery systems and introduction of recombinant genes into visceral tumors by intravascular catheter will extend the application of direct gene transfer to immunotherapy of malignancies. These clinical trials of direct gene transfer will help to develop new treatment strategies for human diseases.

\section{REFERENCES}

1. Price, J., D. Turner \& C. Cepko. 1987. Lineage analysis in the vertebrate nervous system by retrovirus-mediated gene transfer. Proc. Natl. Acad. Sci. USA 84: $156-160$.

2. Nabel, E. G., G. Plautz \& G. J. Nabel. 1990. Site-specific gene expression in vivo by direct gene transfer into the arterial wall. Science 249: 1285-1288.

3. Culver, K. W., Z. Ram, S. Wallbridge, H. Ishit, E. H. Oldfield \& R. M. B I.AESE. 1992. In vivo gene transfer with retroviral vector-producer cells for treatment of experimental brain tumors. Science 256: 1550-1552.

4. Rosenfeld, M. A., W. Siegfried, K. Yoshimura, K. Yoneyama, M. Fukayama, L. E. Stier, P. K. Paakko, P. Gilardi, L. D. Stratford-Perricaudet, M. PerRICAUDET $e t$ al. 1991. Adenovirus-mediated transfer of a recombinant alpha 1antitrypsin gene to the lung epithelium in vivo. Science 252: 431-434.

5. Rosenfeld, M. A., K. Yoshimura, B. C. Trapnell, K. Yoneyama, E. R. Rosenthal, W. Dalemans, M. Fukayama, J. Bargon, L. E. Stier, L. StratrordPerRicaudet et al. 1992. In vivo transfer of the human cystic fibrosis transmembrane conductance regulator gene to the airway epithelium. Cell 68: 143-155.

6. Jaffe, H. A., C. Danel, G. Longenecker, M. Metzger, Y. Setoguchi, M. A. Rosenfeld, T. W. Gant, S. S. Thorgeirsson, L. D. Stratford-Perricaudet, M. Perricaudet, A. Pavirani, J. P. Lecocq \& R. G. Crystal.. 1992. Adenovirus-mediated in vivo gene transfer and expression in normal rat liver. Nature Genet. 1: 372-378.

7. Samul.ski, R. J., X. Zhu, X. XiaO, J. D. Brook, D. E. Housman, N. Etstein \& L. A. HunTER. 1991. Targeted integration of adeno-associated virus (AAV) into human chromosome 19 [published erratum appears in EMBO J. 1992. Mar; 11(3): 1228]. EMBO J. 10: 3941-3950.

8. Palella, T. D., L. J. Silverman, C. T. Schrol.l, F. L. Homa, M. Levine \& W. N. KELLEY. 1988. Herpes simplex virus-mediated human hypoxanthine-guanine phosphoribosyltransferase gene transfer into neuronal cells. Mol. Cell. Biol. 8: 457-460. 
9. Chiocca, E. A., B. B. Chol, W. Z. Cai, N. A. Deluce, P. A. Schaffer, M. DeFiglia, X. O. Breakfield \& R. L. Martuza. 1990. Transfer and expression of the lacZ gene in rat brain neurons mediated by herpes simplex virus mutants. New Biol. 2: 739-746.

10. Breakefield, X. O. \& N. A. DeLuCA. 1991. Herpes simplex virus for gene delivery to neurons. New Biol. 3: 203-218.

11. Wolff, J. A., R. W. Malone, P. Williams, G. Chong, G. Ascadi, A. Jani \& P. FELGNER. 1990. Direct gene transfer into mouse muscle in vivo. Science 247: $1465-1468$.

12. Lin, H., M. S. Parmacek, G. Morle, S. Bolling \& J. M. Leiden. 1990. Expression of recombinant genes in myocardium in vivo after direct injection of DNA. Circulation 82: 2217-2221.

13. Acsadi, G., S. S. Jiau, A. Jani, D. Duke, P. Williams, W. Chong \& J. A. Wolff. 1991. Direct gene transfer and expression into rat heart in vivo. New Biol. 3: 71-81.

14. VILE, R. G. \& I. R. HART. 1993. In vitro and in vivo Targeting of gene expression to melanoma cells. Cancer Res. 53: 962-967.

15. Brigham, K. L., B. Meyrick, B. Christman, M. Magnuson, G. King \& L. C. BERRY. 1989. In vivo transfection of murine lungs with a functioning prokaryotic gene using a liposome vehicle. Am. J. Med. Sci. 298: 278-281.

16. Holt, C. I., N. Garlick \& E. Cornel. 1990. Lipofection of cDNAs in the embryonic vertebrate central nervous system. Neuron 4: 203-214.

17. Plautz, G. E., Z. Y. Yang, B. Wu, X. Gao, L. Huang \& G. J. Nabel. 1993. Immunotherapy of malignancy by in vivo gene transfer into tumors. Proc. Natl. Acad. Sci. USA 90: 4645-4649.

18. Nabel, E. G., Z. Yang, G. Plautz, R. Forough, X. Zhan, C. C. Haudenschit.d, T. MACIAG \& G. J. NABEL. 1993. Recombinant fibroblast growth factor-1 promotes intimal hyperplasia and angiogenesis in arteries in vivo. Nature 362: 844 846.

19. Nabel, E. G., Z. Yang, S. Lip'tay, H. San D. Gordon, C. C. Haudenschil.d \& G. J. NABEL. 1993. Recombinant platelet-derived growth factor B gene expression in porcine arteries induces intimal hyperplasia in vivo. J. Clin. Invest. 91: 1822-1829.

20. Felgner, P. L. \& G. M. Ringold. 1989. Cationic liposome-mediated transfection. Nature 337: 387-388.

21. Felgner, P. L., M. Holm \& H. Chan. 1989. Cationic liposome mediated transfection. Proc. West. Pharmacol. Soc. 32: 115-121.

22. Felgner, P. L., T. R. Gadek, M. Holm, R. Roman, H. W. Chan, M. Wenz, J. P. Northrop, G. M. RINGOLd \& M. Danielsen. 1987. Lipofection: A highly efficient lipid-mediated DNA-transfection procedure. Proc. Natl. Acad. Sci. USA 84: 7413-7417.

23. Nabel, E. G., G. Plautz \& G. J. Nabel. 1992. Transduction of a foreign histocompatibility gene into the arterial wall induces vasculitis. Proc. Natl. Acad. Sci. USA 89: $515 \%-5161$.

24. Lim, C. S., G. D. Chapman, R. S. Gammon, J. B. Muhlestein, R. P. Bauman, R. S. STACK \& J. L. SwaIN. 1991. Direct in vivo gene transfer into the coronary and peripheral vasculatures of the intact dog. Circulation 83: 2007-201l.

25. GAO, X. \& L. HuAng. 1991. A novel cationic liposome reagent for efficient transfection of mammalian cells. Biochem. Biophys. Res. Commun. 179: 280-285.

26. Stewart, M. J., G. E. Plautz, L. Del Buono, Z. Y. Yang, L. Xu, X. Gao, L. Huang, E. G. Nabel \& G. J. Nabel. 1992. Gene transfer in vivo with DNAliposome complexes: Safety and acute toxicity in mice. Hum. Gene Ther. 3: 267-275.

27. Nabel, E. G., D. Gordon, Z.-Y. Xang, L. Xu, H. San, G. E. Pl.autz, X. Gao, L. Huang \& G. J. Nabel. 1992. Gene transfer in vivo with DNA-liposome complexes: Lack of autoimmunity and gonadal localization. Hum. Gene Ther. 3: 649-656.

28. Fearon, E. R., D. M. Pardoli, T. Itaya, P. Golumbek, H. I. Levitsky, J. W. 
Simons, H. Karasuyama, B. Vogelstein \& P. Frost. 1990. Interleukin-2 production by tumor cells bypasses $T$ helper function in the generation of an antitumor response. Cell 60: 397-403.

29. Gansbacher, B., K. Zier, B. Daniels, K. Cronin, R. Bannerji \& E. Gillooa. 1990. Interleukin 2 gene transfer into tumor cells abrogates tumorigenicity and induces protective immunity. J. Exp. Med. 172: 1217-1224.

30. Thepper, R. I., P. K. Pattfngai.e \& P. Lfeder. 1989. Murine interleukin-4 displays potent antitumor activity in vivo. Cell 57: 503-512.

31. Li, W., T. Diamantstein \& T. Blankenstein. 1990. Lack of tumorigenicity of interleukin 4 autocrine growing cells seems related to the anti-tumor function of interleukin 4. Mol. Immunol. 27: 1331-1337.

32. Golumbek, P. T., A. J. Lazenby, H. I. Levitsky, L. M. Jaffee, H. Karasuyama, M. Baker \& D. M. Pardoll. 1991. Treatment of established renal cancer by tumor cells engineered to secrete interleukin-4. Science 254: 713-716.

33. Gansbacher, B., R. Bannerji, B. Daniels, K. Zier, K. Cronin \& E. Gilboa. 1990. Retroviral vector-mediated $\gamma$-interferon gene transfer into tumor cells generates potent and long lasting antitumor immunity. Cancer Res. 50: 7820-7825.

34. Chen, L., S. Ashe, W. A. Brady, I. Hellstrom, K. E. Hellstrom, J. A. LedbetTER, P. MCGOWAN \& P. S. LINSI.EY. 1992. Costimulation of antitumor immunity by the B7 counterreceptor for the T lymphocyte molecule CD28 and CTLA-4. Cell 71: 1093-1102.

35. TownSEnd, S. \& J. P. Allison. 1993. Tumor rejection after direct costimulation of CD8 + T cells by B7-transfected melanoma cells. Science 259: 368-370.

36. Blankenstein, Th., Z. Qin, K. ÜBerla, W. Mulller, H. Rosen, H.-D. Vol.k \& T. DiAMANSTEIN. 1991. Tumor suppression after tumor cell-targeted tumor necrosis factor a gene transfer. J. Exp. Med. 173: 1047-1052.

37. Teng, M. N., B. H. Park, H. K. Koepren, K. J. Tracey, B. M. Fendly \& H. SCHR EIBER. 1991. Long-term inhibition of tumor growth by tumor necrosis factor in the absence of cachexia or T-cell immunity. Proc. Natl. Acad. Sci. USA 88: 3535-3539.

38. Asher, A. L., J. J. Mulé, A. Kasid, N. P. Restifo, J. C. Salo, C. M. Reichert, G. Jafie, B. Fendi.y, M. KRiegler \& S. A. Rosenberg. 1991. Murine tumor cells transduced with the gene for tumor necrosis factor- $\alpha$. J. Immunol. 146: 32273234.

39. Parham, P., C. E. Lomen, D. A. Lawlor, J. P. Ways, N. Holmes, H. L. Coppin, R. D. Salter, A. M. WAN \& P. D. EnNis. 1988. Nature of polymorphism in HLA-A, -B, and -C molecules. Proc. Natl. Acad. Sci. USA 85: 4005-4009.

40. SKINNER, M. A. \& J. MARBROOK. 1976. An estimation of the frequency of precursor cells which generate cytotoxic lymphocytes. J. Exp. Med. 143: 1562-1567.

41. LINDAHL, K. F. \& D. B. WILSON. 1977. Histocompatibility antigen-activated cytotoxic T lymphocytes I. Estimates of the absolute frequency of killer cells generated in vitro. J. Exp. Med. 145: 500-522.

42. Wahi, W. L., G. E. Plautz, B. A. Fox, G. J. Nabei, S. Shu \& A. E. Chang. 1992. Generation of therapeutic lymphocytes after in vivo transfection of tumor with a gene encoding allogeneic class I MHC antigen. Surg. Fonum 63: 476-478.

43. Nabel, G. J., A. Chang, E. G. Nabel, G. Plautz, B. A. Fox, L. Huang \& S. Shu. 1992. Clinical protocol. Immunotherapy of malignancy by in vivo gene transfer into tumors. Hum. Gene Ther. 3: 399-410.

44. Nabel, G. J., E. G. Nabel, Z. Yang, B. Fox, G. Plautz, X. Gao, L. Huang, S. Shu, D. Gordon \& A. E. Chang. 1993. Direct gene transfer with DNA liposome complexes in humans: Expression, lack of toxicity and therapeutic potential in melanoma. Proc. Natl. Acad. Sci. USA 90: 10759-10763. 